\title{
Lattice and Schottky contributions to the morphology of lanthanide heat capacities
}

\author{
EDGAR F. WESTRUM. Jr. \\ Department of Chemistry. The University of Michigan. \\ Ann Arbor, MI 48109, U.S.A. \\ Being the third \\ ROSSINI LECTURE \\ of the Commission of Thermodynamics of the International Union of \\ Pure and Applied Chemistry. \\ delivered on 10 September 1982 \\ at its Conference on Chemical Thermodynamics held at \\ University College London. U.K.
}

\section{Introduction}

As many of you will already know, my research interests have largely centered on the utilization of heat-capacity measurements with adjuvant quantities from other sources when needed or when feasible -in the solution of scientific problems of many sorts. Often this interest was triggered by some fascinating transition or other, but whatever the scientific motivation we have almost invariably used that interest to provide chemical-thermodynamic values for use in science and technology. For these reasons and despite the fact that measurements were often tedious, at times frustratingly laden with hysteresis, and quite painstaking, the adventure is becoming more exciting in that we have computerized the measurements enabling us to do a great many more determinations, with even higher accuracy and precision, and at the same time to undertake investigations on a broader scale than when all of the work was done around the clock by a pair of scientists. These investigations led us into hydrogen bonding, actinides and reactor safety, lanthanides, transition-element pnictides and chalcogenides, plastically-crystalline molecular crystals, $\pi-\pi$ molecular complexes, intermetallic compounds, and even a few metals. At the moment our interest is concerned with samples which come from mineralogy and petrology; their behavior is fully as complex as one might expect when dealing with magnetic. electronic, and the great variety of named transitions which one encounters in inorganic chemistry.

Among the most exciting events in the determination of solid-state heat capacities is the occurrence of a "transition"-be it phase or otherwise. Whether one describes 
this thermodynamically as a crisis in the Gibbs energy-or microscopically in terms of molecular or ionic freedom -the resultant phenomenon does seem to be totally disproportionate to the very subtle "causes" involved. On a recent visit to the People's Republic of China I learned the expression "observing flowers from horseback": since time does not permit a thorough treatment of all the interesting facets involved, we shall gallop through and focus mainly on the Schottky contribution to heat capacity - mostly for its inherent interest-but also in a non-trivial way to utilize it as a tool to deduce the reliability of a scheme for the evaluation of the lattice contribution of the heat capacity-an essential step in analyzing the morphology of the heat-capacity curve.

\section{The Schottky contribution to heat capacity}

\section{THE CONTRIBUTION ITSELF}

I am confident that the participants in this Conference are all well acquainted with the nature of a Schottky function; one would only wish that the same could be said for physical chemists in general. Although the name of Werner Schottky is undoubtedly a household word among physical chemists, usually it is referred to in connection with the Schottky effect or the Schottky defect, but more than half a century ago he also wrote a single paper on a third phenomenon often called the Schottky anomaly. (1) This anomaly will be referred to as the Schottky contribution and presented mainly in terms of the heat capacity. It does occur in many other thermophysical chemical properties, for example the thermal expansion of a substance. In its simplest form it represents simply the presence of an excited electronic level in a substance. Each level is characterized by a certain degeneracy $g_{i}$. The occurrence of this contribution at sufficiently low temperatures gives rise to a characteristic bell-shaped curve skewed out on the high-temperature side. The temperature of the peak is related to the energetic separation of the pair of levels and the maximum height is determined by the ratio of the degeneracies. Moreover, the total entropy under the curve is also related to the number of levels and the degeneracies involved. When the electronic energy increments are sufficiently small the Schottky anomaly occurs at a temperature at which the lattice contribution is so small that the Schottky contribution stands out as an obvious bell-shaped anomaly. When the energy levels are sufficiently spread to cause the occurrence of the contribution above the ambient temperature, the maximum is typically so spread out as to be unrecognized unless the heat capacities of isostructural substances with nearly identical molar masses are compared. Nonetheless, it does represent an important aspect of the morphology of the heatcapacity curves and tends to do so increasingly as higher temperatures are involved whenever one is dealing with transition-element, lanthanide-element, and actinideelement compounds. It has always seemed strange to me that Schottky-despite abundant fame from his effect and defect-never again, so far as I have been able to ascertain, referred to this anomaly, not even in the renowned textbook Thermodynamik which he co-authored with Ulich and Wagner. Unfortunately, few modern textbook authors either recognize or mention the great significance of the Schottky anomaly vis-à-vis heat-capacity curves. 
Although none of the examples Schottky gave in his original paper were indeed other than poor heat-capacity measurements and not true examples of Schottky contributions, real Schottky contributions have been measured on occasion since the early 1930 's by calorimetrists, most often on magnetically dilute materials. Virtually all of those that have been measured subsequently do not seem to agree with the heatcapacity anomaly calculated from the spectroscopically determined energy levels themselves within the combined uncertainties.

\section{BRIEF MATHEMATICAL INTERLUDE}

The Schottky heat-capacity function $\operatorname{Sch}(u)$ is rigorously related to Einstein heatcapacity functions $\operatorname{Ein}(u)$ by both an additive and a multiplicative identity:

and

$$
\operatorname{Ein}(u)-\operatorname{Ein}(2 u)=\operatorname{Sch}(u)
$$

$$
\operatorname{Ein}(u) \operatorname{Sch}(u)=u^{2} \operatorname{Ein}(2 u)
$$

This is by no means the limit of the rigorous relations; in the course of considerable experimentation with the mathematics of these functions we have deduced 40 or 50 relations of which only several will be presented here. I should note that these are restricted to two-level Schottky functions with equal degeneracies and at the present time to integral arguments. For the more difficult-and far more useful-cases of many levels, differencing degeneracies, and arguments which are not integers but which differ from one another by, say, a per cent or so, we have been obliged to use series approximations or numerical evaluation and somewhat less rigor. This is a bit disconcerting since some of my friends claim to find evidence for Schottky functions by differencing the heat capacities of a compound and its diamagnetic analog utilized to represent a lattice contribution. In these instances the difference is indeed probably exactly a Schottky function, but it is occasioned simply by the fact that the Einstein or Debye $\Theta_{\mathrm{S}}$ of the two substances differ slightly, and the apparent Schottky contribution is an artifact occasioned by the additive identity already presented.

Moreover, the difference in the heat-capacity of a vitreous phase and the corresponding crystal (e.g. $\left.\mathrm{B}_{2} \mathrm{O}_{3}\right)$ is also-for somewhat the same reasons-a Schottky function. ${ }^{(2)}$

\section{A WORD ON MORPHOLOGY}

The Schottky function is a beast that is little hard to "fudge". Its shape is totally determined by the levels and the degeneracies. Maximum height is independent of the temperature and governed only by the degeneracies in the simple system already discussed. In a system with more energy levels, the shape of the anomaly is determined of course by the overall pattern of energy levels, and the calculations made, for the simple case of only two levels, by the equation:

$$
C_{\text {Schotiky }} / R=u^{2}\left(g_{0} / g_{1}\right) \exp (u) /\left\{1+\left(g_{0} / g_{1}\right) \exp (u)\right\}^{2}: \quad\left(u=e_{1} / k T\right) .
$$

It is interesting to note in passing that the Schottky function can also be represented 
by the difference between the squared a verage energy of the system and the average of the square of the mean energy itself as shown in the equation:

$$
C_{\text {Schotky }} / R=\left(\langle E\rangle^{2}-\left\langle E^{2}\right\rangle\right) /(R T)^{2},
$$

or for a two-level equidegenerate system

$$
C_{\text {Schotiky }} / R=\{(u / 2) \operatorname{sech}(u / 2)\}^{2} .
$$

(a simple symmetrical formula designed to obfuscate the reader).

It is interesting to see that in the case of a multi-level system $\left(\mathrm{PrCl}_{3}\right.$, figure 1) the energy levels separate into two distinct peaks.

Very often one encounters a need to appreciate the morphology of a three-level system and these curves are shown in figure 2 for a variety of values of the ratio of energies between the two levels: i.e. for values of $X=\left(E_{1}-E_{0}\right) /\left(E_{2}-E_{0}\right)$.

Other interesting aspects of the morphology of Schottky and Schottky-like contributions have been discussed elsewhere. (3)

\section{Early studies of the lanthanide sesquioxides}

Recognition of the importance of the Schottky thermophysical contribution came with a series of papers by Justice and Westrum ${ }^{(4-8)}$ who studied the lanthanide sesquioxides and obtained an unusually rich yield of results concerning the energetics of the trivalent ions in these compounds. Although the Schottky contributions may also be studied spectroscopically, the general unavailability of single-crystal samples for absorption spectroscopy or for paramagnetic-resonance experiments had tended to favor the calorimetric approach. Our initiatory measurements ${ }^{(4)}$ on neodymium sesquioxide yielded results in levels more than 10 times smaller than those estimated by Penney ${ }^{(9)}$ from crystal-field splittings. Rut any discredit was of short duration,

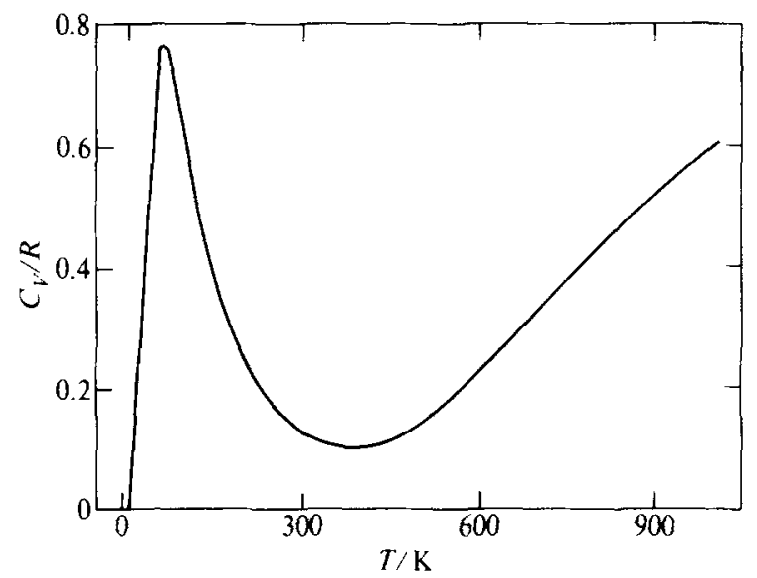

FlGURE 1. The Schottky heat capacity of $\mathrm{PrCl}_{3}$ (in part) based on the wavenumbers (and their degeneracies): 33.5 (1), 102 (2), 148 (2), 163 (1), 228 (1). 2134 (1). 2168 (2), 2190 (2). 2208 (1). 2230 (2), $2260(2)$, and $2280(2) \mathrm{cm}^{-1}$. 


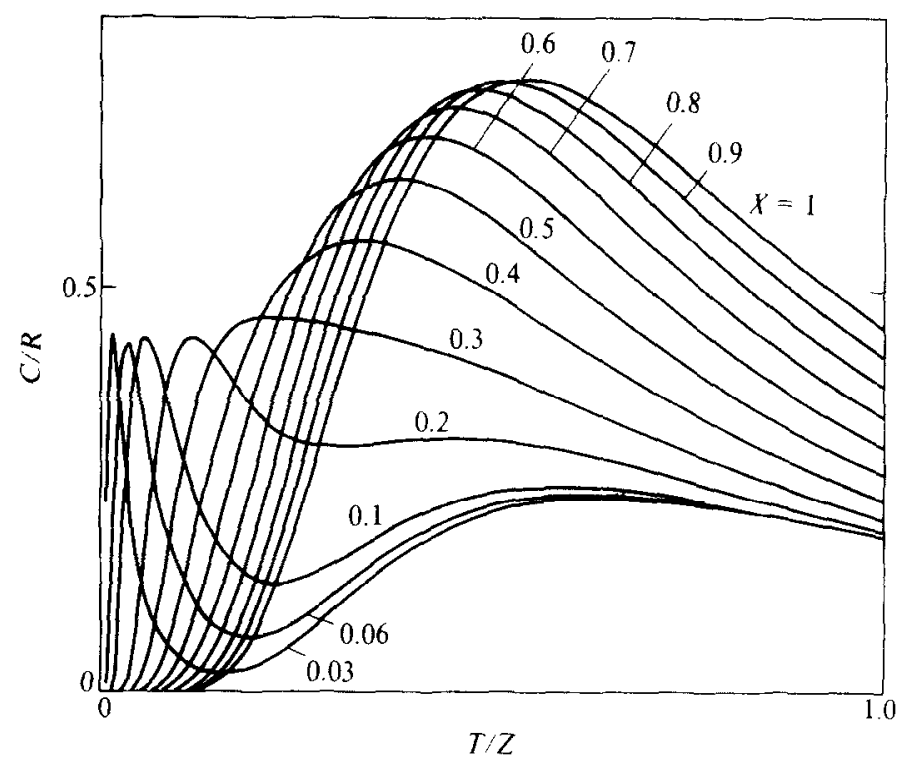

FIGURE 2. Schottky functions for 3 equidegenerate-level systems related to the parameters: $X=\left(E_{1}-E_{0}\right) /\left(E_{2}-E_{0}\right)$ and $\mathcal{Z}-\left(E_{2}-E_{0}\right)$.

since spectroscopy ${ }^{(10)}$ confirmed, in this instance, the values that had been obtained by calorimetry. The quantitative comparison is made in table 1 .

The method of approach involved measurement of the total heat capacity of $\mathrm{Nd}_{2} \mathrm{O}_{3}$, of a diamagnetic analog $\mathrm{La}_{2} \mathrm{O}_{3}$, and the resolution of the difference in heat capacity of the two compounds in terms of a sequence of Schottky levels of the degeneracies predicted by crystal-field theory. The power of the cryogenic calorimetric approach thus demonstrated was later extended by the same authors to include most other lanthanide sequioxides, even those containing $C_{2}$ and $C_{3 i}$ sets of levels by adjustment of the lattice heat capacity as described later. It was demonstrated that the levels were valid not only for the cryogenic heat-capacity contribution, but as well for temperatures in excess of $1000 \mathrm{~K}$. Unfortunately, the sesquioxides crystallized in A-, B-, and $C$-forms thus making it harder to recognize the underlying trends. A recent paper extends this fitting and correlation to new electronic Raman scattering results ${ }^{(11)}$ and is discussed later.

TABLE 1. Stark wavenumbers $\sigma$ for $\mathrm{Nd}_{2} \mathrm{O}_{3}$

\begin{tabular}{|c|c|c|c|c|}
\hline Level & $y_{i}^{u}$ & Calc. $^{h}$ & $\begin{array}{c}\sigma / \mathrm{cm}^{-1} \\
\text { Calorimetry }\end{array}$ & Spectroscopy \\
\hline 0 & 2 & 0 & 0 & 0 \\
\hline 1 & 2 & 492 & 21 & 22 \\
\hline 2 & 2 & 1476 & 81 & 8.3 \\
\hline 3 & 2 & 2952 & 400 & 390 \\
\hline 4 & 2 & 4920 & 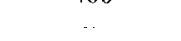 & $\cdots$ \\
\hline
\end{tabular}

${ }^{a}$ Degeneracy of level. ${ }^{b}$ Penney, reference 9. 'Justice and Westrum. reference 4. ${ }^{d}$ Henderson et $a l$, reference 10 . 


\section{Other interesting lanthanide compounds}

\section{LANTHANIDE TRICHLORIDES}

The desirability of extending such studies to other systems led Sommers and Westrum to examine the lighter lanthanide trichlorides. ${ }^{(12.13)}$ Their study further heightened the understanding of the trend and regularities involved and showed the importance of the Schottky contribution to the thermophysical functions. The latter were in excellent accord with those predicted by the scheme of Westrum ${ }^{(14)}$ based upon the treatment of Gronvold and Westrum. ${ }^{(15)}$ The quality of the accord can be seen in table 2 .

Lanthanide pnictides. The heat capacities of a set of lanthanide mononitrides have been determined by Stuttius ${ }^{(16)}$ on materials less well characterized than desirable. We are presently undertaking a reinterpretation of these results. His work is interesting in that he did utilize crystal parameters in interpolating lattice heat capacities across the lanthanide series.

\section{LANTHANIDE HEXABORIDES}

Several of these fascinating compounds have been studied over the cryogenic range by Westrum et al.;(17) these studies are interesting and will be examined.

\section{Better lattice-contribution evaluation}

Before we consider other lanthanide systems we should note that the oxides have relatively smaller variation in their lattice contributions than do some of the other lanthanide series. As has already been noted. Schottky contributions can be deduced also from spectroscopic observations (or from theoretically evaluated crystal-field

TABLE 2. Comparison of some trichloride entropy estimation schemes $\left(R=8.314 \mathrm{~J} \cdot \mathrm{K}^{-1} \cdot \mathrm{mol}^{-1}\right)$

\begin{tabular}{|c|c|c|c|c|}
\hline \multirow[b]{2}{*}{ Compound } & \multirow[b]{2}{*}{ Latimer } & $\multicolumn{2}{|c|}{S(298.15 \mathrm{~K})-S(0)} / R$ & \multirow[b]{2}{*}{ Experimental $]^{(12.13)}$} \\
\hline & & $\begin{array}{c}\text { Latimer } \\
\text { augmented a }\end{array}$ & $\begin{array}{c}\text { Westrum } \\
\text { augmented }\end{array}$ & \\
\hline $\mathrm{LaCl}_{3}$ & 17.4 & 17.4 & 16.5 & 16.54 \\
\hline $\mathrm{CeCl}_{3}$ & 17.4 & 19.2 & 18.1 & $(18.1)^{\circ}$ \\
\hline $\mathrm{PrCl}_{3}$ & 17.4 & 19.6 & 18.5 & $18.44^{\circ}$ \\
\hline $\mathrm{NdCl}_{3}$ & 17.3 & 19.7 & 18.5 & 18.45 \\
\hline $\mathrm{PmCl}_{3}$ & 17.5 & 20.0 & 18.5 & $(18.6)^{\circ}$ \\
\hline $\mathrm{SmCl}_{3}$ & 17.5 & 19.3 & 18.0 & 18.06 \\
\hline $\mathrm{EuCl}_{3}$ & 17.5 & 18.8 & 17.4 & 17.32 \\
\hline $\mathrm{GdCl}_{3}$ & 17.6 & 19.7 & 18.1 & 18.21 \\
\hline
\end{tabular}

${ }^{a}$ By $R \ln (2 J+1)$; the $\left(\mathrm{Cl}_{3}\right)^{3-}$ ion contribution is taken as $43.5 \mathrm{~J} \cdot \mathrm{K}^{-1} \cdot \mathrm{mol}^{-1}$

${ }^{b}$ By $R \ln (2 \mathrm{~J}+1)$; the $\left(\mathrm{Cl}_{3}\right)^{3-}$ ion contribution is taken as $37.7 \mathrm{~J} \cdot \mathrm{K}^{-1} \cdot \mathrm{mol}^{-1}$.

- Parentheses denote interpolated lattice and calculated Schottky contributions.

Based on $0.294 \mathrm{~K}$. 
parameters) and a comparison made between such a "spectroscopic" Schottky contribution and a "calorimetrically" deduced Schottky contribution-but to do so requires resolution of the Schottky contribution from the observed total heat capacity. This requires that we have a suitable scheme to deduce the lattice heatcapacity contribution. A suitable scheme has not been available so in this section we discuss the utilization of such a comparison-first to test the reliability of a particular scheme and second to obtain the resolution of Schottky functions by this so-called "volumetric scheme".

Because the heat capacity of a system is determined by the energy levels of that system, heat-capacity measurements themselves are often used to deduce those energy levels. Very often one has a model of a system and can calculate the resultant excess heat capacity associated with the model. Unfortunately, the heat capacity of interest is usually superimposed on a background contribution and the separation of the excess contribution must be made from that of the background. All too often, this resolution is made by "good judgment" only. The relevant background contribution is usually the so-called lattice contribution and inasmuch as it typically represents 80 to 100 per cent of the total measured heat capacity. if it is going to be estimated, the procedure must be one of relatively high accuracy. Consequently, whenever resolution of transitions (be they magnetic, structural, Schottky, order-disorder) is to be done, one is confronted with the necessity of an evaluation of the lattice contribution. When estimates are made of the heat capacity and/or the thermodynamic functions of substances, the lattice represents the bulk of the quantity. Moreover, when adjustment of thermophysical properties, e.g. to endmember composition of mineralogical or petrological materials, is involved, the same problem arises. Consequently, an urgent need exists for evaluation of the lattice contribution both for resolution and for prediction.

We consider that we have recently made limited - but none the less-significant progress in this matter and that the volume of the crystal over an important portion of the temperature scale is clearly involved. Nonetheless, mass, coordination number. and structure-type are also relevant quantities and will need to be invoked ultimately.

\section{THE GRONVOLD-WESTRUM ENDEAVOR}

The story begins with the 1961 Gronvold and Westrum paper on the correlation of entropies for transition-element chalcogenides ${ }^{(15)}$ and the graphical representation of the surprising result of preparation for a 1977 Moscow plenary lecture ${ }^{(18.19)}$ in which the correlation was compared with the Latimer ${ }^{(20.21)}$ scheme for entropy contributions for cations as shown in figure 3. Latimer considered that these contributions were proportional to the logarithm of the mass of the cation. Two aspects of our findings surprising to me were

- The "main stream"-3d-, 4d-, and 5d-transition-element-cations almost all show the same contribution to the cationic entropy contribution rather than a Latimer-like mass dependence. ${ }^{(20.21)}$ Borderline transition-element chalcogenides are possibly intermediate. 
- The entropy values for 4f (lanthanide) cations are essentially diametrically perpendicular to the mass dependence predicted by the Latimer scheme suggesting that although masses and volumes change by roughly the same fractional magnitude through the system-but in the opposite sense--the heatcapacity trend here clearly favored volume rather than mass dependence.

On the basis of these notions we studied lanthanide systems; first the trichlorides, $\mathrm{LnCl}_{3},{ }^{(12.13,22)}$ and then the trihydroxides $\mathrm{Ln}(\mathrm{OH})_{3},{ }^{(23-28)}$ which have the great advantages of being isostructural throughout the series and having larger latticecontributions.

In the discussion which follows, frequent reference is made to Schottky contributions-interesting in their own right - but used here initially and primarily as a means of testing the success of the evaluation of the lattice contribution.

\section{OTHER INTERPOLATION SCHEMES FOR HEAT CAPACITIES AND ENTROPIES}

In principle, the best way to evaluate $C_{\text {lat }}$ is from the phonon dispersion relation $K(\omega)$ determined by inelastic neutron scattering. However, occasionally Einstein functions (in which $\omega_{\mathrm{E}}=$ constant) or Debye functions (in which $\omega_{\mathrm{D}}=\gamma_{0} k$ ) can be used to get results reliable within about 10 per cent.

$\Lambda$ ttempts to get an experimental estimate of $C_{\text {lat }}$ in compound $X$ by measuring the heat capacity of an isostructural diamagnetic compound ID are frequent. Here the corresponding states assumption:

$$
C_{\text {latt }}(\mathrm{X}) T=C_{\text {lati }}(\text { ID }) k T \text {, }
$$

in which $k$ is experimentally deduced, is often employed.

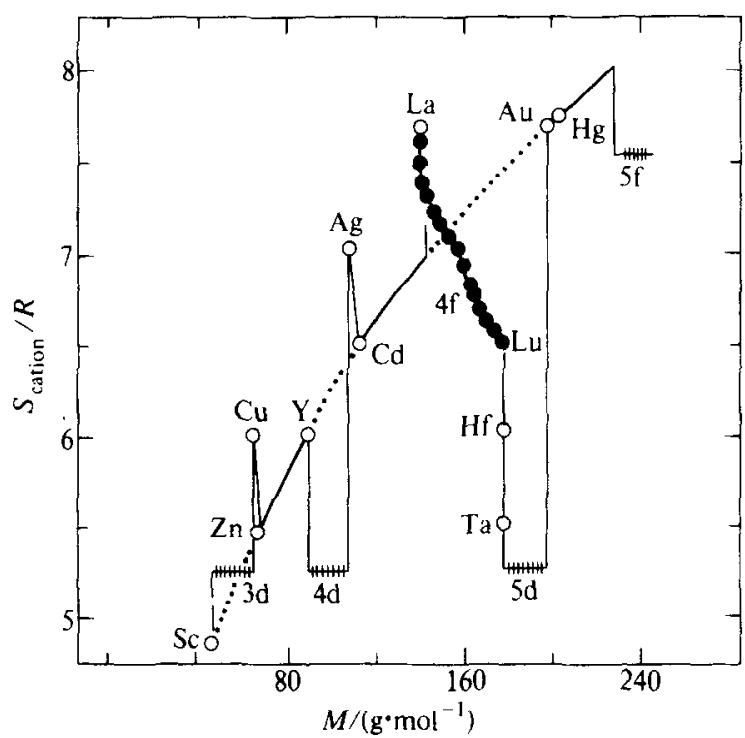

FIGURE 3. Comparison of Grønvold and Westrum's scheme ${ }^{(151}$ against the background of the Latimer scheme. ${ }^{(20.21)} \cdots, \bigcirc$. The Latimer-scheme contributions;, experimental values for the lanthanide cations: ${ }^{(14)} \ldots$, experimental values for the transition-element cations. 
Alternatively, the Debye $\Theta_{\mathrm{D}}$ approximation may be couched in the mass $m$ of the molecules:

$$
\Theta_{\mathrm{D}}(\mathrm{X}) / \Theta_{\mathrm{D}}(\mathrm{ID})=\{m(\mathrm{ID}) / m(\mathrm{X})\}^{1 / 2} .
$$

The more refined Lindemann relation using melting temperatures $T_{\mathrm{f}}$ and molar volumes $V_{\mathrm{m}}$ is also used

$$
\Theta_{\mathrm{D}}^{2}=k^{\prime} T_{\mathrm{f}} / m V_{\mathrm{m}}^{2 / 3} .
$$

Corresponding-states approaches are often used; but for nearly half a century the Latimer scheme ${ }^{(20.21)}$ has been a favorite way of taking into account the differences between compounds in iso-anionic series. This time-honored scheme-devised primarily for entropy estimates--is not without its flaws, despite the several times it was adjusted by Latimer himself.

\section{THE VOLUMETRIC SCHEME FOR HEAT CAPACITIES}

The scheme that we have advocated involves linear interpolation on the basis of the molar volumes of the compounds in question. In particular, the formula by which the lattice heat capacity of the praseodymium trihydroxide may be calculated is

$$
C_{p}\left\{\operatorname{Pr}(\mathrm{OH})_{3} \text {, lattice }\right\}=x C_{p}\left\{\mathrm{La}(\mathrm{OH})_{3}\right\}+(1-x) C_{p}\left\{\mathrm{Gd}(\mathrm{OH})_{3}\right\} .
$$

and in which $x$ is the fractional molar volume increment, i.e.

$$
x=\left[V_{\mathrm{m}}\left\{\operatorname{Pr}(\mathrm{OH})_{3}\right\}-V_{\mathrm{m}}\left\{\mathrm{La}(\mathrm{OH})_{3}\right\}\right] /\left[V_{\mathrm{m}}\left\{\mathrm{Gd}(\mathrm{OH})_{3}\right\}-V_{\mathrm{m}}\left\{\mathrm{La}(\mathrm{OH})_{3}\right\}\right] .
$$

It should be noted that utilization of other than linear interpolations would have involved differences in only second-order effects. The importance of volume was appreciated $^{(\mathbf{1 8 . 1 9 )}}$ on recognition of the fact that for the lanthanide chalcogenides the lattice contribution decreased with increasing atomic number and was, therefore, diametrically opposed to the trend in the Latimer scheme based on mass. The lanthanide contraction provided the clue (figure 3). Other authors ${ }^{(29.30)}$ have been engaged in a polemic as to the relevance of volume against mass in providing interpolation schemes for lattice contributions. Moreover, Kieffer ${ }^{(31-35)}$ has undertaken a theoretical and experimental correlation of the lattice vibrations of minerals. This takes into account the many factors involved and discusses particularly the analysis of the vibrational contribution. which has been discussed also by Sommers and Westrum. ${ }^{(13)}$

But does the volumetric scheme really work? Perhaps the best way of testing the validity of a lattice-contribution scheme is in the calculation of the calorimetric Schottky contribution and the comparison of this excess heat capacity with that calculated from spectroscopic quantities on the samples themselves. However, this comparison can be made only when one utilizes the Stark levels of the concentrated compounds. Measurements made on doped lanthanide halides, for example, need to be extrapolated by some technique-discussed elsewhere ${ }^{(22)}$ - or by calculations based on crystal-field parameters. 


\section{SOME TESTS OF THE SCHEME ON LANTHANIDE TRIHYDROXIDES AND TRICHLORIDES}

Since resolution of Schottky contributions from the generally much larger vibrational (lattice) heat capacities of lanthanide compounds has been limited by the uncertainty in the magnitude of the lattice contribution, such subtle effects as dependence of the Stark levels on temperature and host lattice have been heretofore undetected calorimetrically.

Since the lanthanide trihydroxides are an iso-anionic series having relatively small lattice contributions and their lower-lying Stark levels have been spectroscopically deduced for many of the concentrated compounds, this series is the most nearly ideal system we have yet studied in an attempt to resolve Schottky contributions in the 5 to $350 \mathrm{~K}$ range. Threc examples illustrate the success of the scheme described on $\mathrm{Ln}(\mathrm{OH})_{3}$ systems; moreover, two examples from $\mathrm{LnCl}_{3}$ systems demonstrate that excellent agreement obtains here as well.

$\mathrm{Eu}(\mathrm{OH})_{3}$. The Schottky contribution to the heat capacity of the Eu(III) analog is unique in that it arises entirely from thermal populations of excited [SL] $J$-manifolds. This invariably results in the lowest excited Stark levels being much higher in energy for the Eu(III) analog than for any other series member. The calculated Schottky heat capacity is consequently relatively insensitive to small shifts in the Stark energies and, therefore, is expected to be the most accurate approximation to the true Schottky heat capacity within any lanthanide series.

The energy levels of concentrated $\mathrm{Eu}(\mathrm{OH})_{3}$ were determined by Cone and Faulhaber $^{(36)}$ from absorption and fluorescence spectra at 4.2 and $7.7 \mathrm{~K}$. Stark levels arising from the ${ }^{7} F_{0},{ }^{7} F_{1},{ }^{7} F_{2}$, and ${ }^{7} F_{3}$ manifolds all contribute to the Schottky heat capacity below $350 \mathrm{~K}$. The derived calorimetric Schottky contribution shown in figure 4 is seen to be in excellent accord with that calculated from the spectroscopic quantities. $^{(24)}$

$\operatorname{Pr}(\mathrm{OH})_{3}$. The crystal-field splitting of the ${ }^{3} \mathrm{H}_{4}$ manifold of $\operatorname{Pr}(\mathrm{OH})_{3}$ has been determined from the absorption spectra of mulls at $95 \mathrm{~K} .{ }^{(37)}$ The observed spectra were not as highly resolved as one might obtain from measurements on single crystals. This lack of resolution is reflected in $\mathrm{a} \pm 3 \mathrm{~cm}^{-1}$ uncertainty in the Stark wavenumbers. As seen in figure 4 the calorimetric and spectroscopic Schottky curves are in very good agreement between 15 and $230 \mathrm{~K}$. Below $25 \mathrm{~K}$ a cooperative magnetic contribution of unknown magnitude plus the uncertainty in the energy of the lowest excited Stark level preclude any attempt to determine the Schottky contribution accurately in this temperature region. Above $260 \mathrm{~K}$ the calorimetric curve trends below the calculated band. Such a decrease in the high-temperature calorimetric Schottky curve could be due to a gradually decreasing crystal-field intensity within the $\operatorname{Pr}(\mathrm{OH})_{3}$ crystals as the lattice expands with temperature. (A gradual shift to lower wavenumbers of 5 to $10 \mathrm{~cm}^{-1}$ by the four highest Stark components of the ${ }^{3} \mathrm{H}_{4}$ manifold between 100 and $350 \mathrm{~K}$ would account for the observed deviation.)

$\mathrm{Tb}(\mathrm{OH})_{3}$. The energy levels of the lowest four manifolds of concentrated $\mathrm{Tb}(\mathrm{OH})_{3}$ and $\mathrm{Tb}^{3+}$-doped $\mathrm{Y}(\mathrm{OH})_{3}$ were determined by Scott, Meissner, and Crosswhite. ${ }^{(38)}$ 


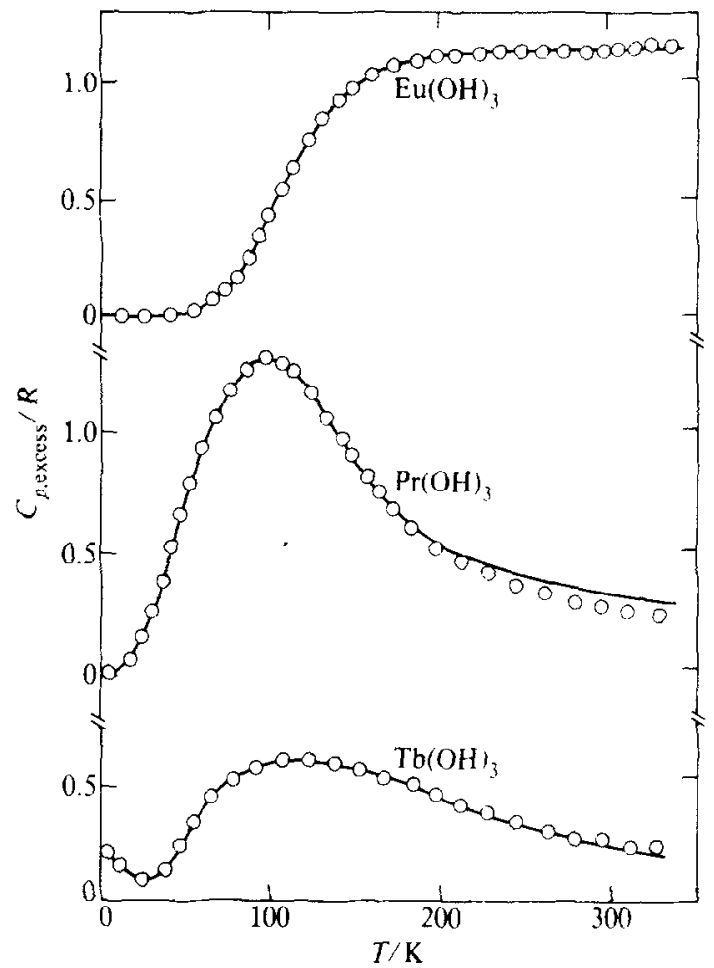

FIGURE 4. Schottky heat-capacities for several lanthanide trihydroxides. - - The spectroscopic Schottky contributions: $O$, the calorimetric Schottky contributions resolved by the volumetric method.

The observed Schottky anomaly below $350 \mathrm{~K}$ is due almost entirely to population of the ${ }^{7} \mathrm{~F}_{6}$ manifold. The availability of spectroscopically determined energy levels for both the $\mathrm{Tb}(\mathrm{OH})_{3}$ and $\mathrm{Y}(\mathrm{OH})_{3}$ host lattices provides an opportunity to observe directly the sensitivity of the new lattice-contribution approximation technique in differentiating between such systems. Heretofore the general assumption has been that any calorimetrically derived Schottky contribution is too crude to detect the effect of any differences in the Stark level energies of such systems.

As seen in figure 4 the calorimetric and calculated $\mathrm{Tb}(\mathrm{OH})_{3}$ Schottky curves are in excellent agreement below $160 \mathrm{~K}$, while at higher temperatures the calorimetric curve trends below that deduced from the spectral quantities. ${ }^{(26)}$ The calorimetric Schottky curve is clearly in far better agreement with the spectroscopic curve calculated from the Stark levels of concentrated $\mathrm{Tb}(\mathrm{OH})_{3}$ rather than with that of $\mathrm{Tb}^{3+}$-doped $\mathrm{Y}(\mathrm{OH})_{3}$. (Above $160 \mathrm{~K}$ the difference between the calorimetric and calculated curves may be accounted for if the Stark levels are assumed to undergo an approximately 6 per cent shift to lower energies between 77 and $350 \mathrm{~K}$. Such a shift may be postulated to be occasioned by the decrease of the crystal-field intensity as the lattice expands with increasing temperature.)

$\mathrm{EuCl}_{3}$. The Schottky heat-capacity contribution of $\mathrm{EuCl}_{3}$ is unique--like that of 
$\mathrm{Eu}(\mathrm{OH})_{3}$-in that it arises entirely from thermal population of excited [SI] $J$-manifolds. The first excited states are near 355 and $405 \mathrm{~cm}^{-1}$. The Schottky contribution was calculated from energy levels of ( 1 and 4 per cent) $\mathrm{Eu}^{3+}$-doped $\mathrm{LaCl}_{3}$ determined from the absorption spectrum at $4 \mathrm{~K}$ and the fluorescence spectrum at 4 and $77 \mathrm{~K}$ studied by Deshazer and Dieke. The energy levels of concentrated $\mathrm{EuCl}_{3}$ are not expected to be identical to those of $\mathrm{Eu}^{3+}$-doped $\mathrm{LaCl}_{3}$. The stronger crystal field in concentrated $\mathrm{EuCl}_{3}-$ compared with that in the $\mathrm{LaCl}_{3}$ host -is expected to increase the Stark splitting and simultaneously to lower the center of gravity of the ${ }^{7} \mathrm{~F}_{1}$ manifold, i.e. to lower the energy of the $\mu=1$ doublet and to leave the $\mu=0$ level essentially unchanged. The effect of the stronger crystal field will be countered to some extent by expansion of the $\mathrm{EuCl}_{3}$ lattice at higher temperatures (i.e. in the region of the Schottky maximum); however, this is anticipated to be insufficient fully to nullify the effect. Because the wavenumbers of the Stark levels contributing to the Schottky heat capacity are relatively high, a shift of the $\mu=1$ doublet by as much as 10 to $15 \mathrm{~cm}^{-1}$ will have but a small effect on the calculated Schottky contribution. The derived calorimetric Schottky heat capacity shown in figure 5 is seen to be in excellent accord with that derived from the spectroscopic quantities. ${ }^{(2)}$

$\mathrm{PrCl}_{3}$. The analysis of the Schottky contribution to the heat capacity of $\mathrm{PrCl}_{3}$ is complicated by unusual shifts in the Stark energies as the intensity of the crystalline field is varied. However, the basic arguments remain essentially unchanged from those applied to the preceding compounds. The energy levels of $\mathrm{Pr}^{3+}$-doped $\mathrm{LaCl}_{3}$ were determined from absorption and fluorescence spectra by Sarup and Crozier. Although the wealth of spectroscopic data may be best deduced by reference to the definitive paper, ${ }^{(22)}$ both figure 5 and table 3 attest to the good agreement between the two Schottky contributions.

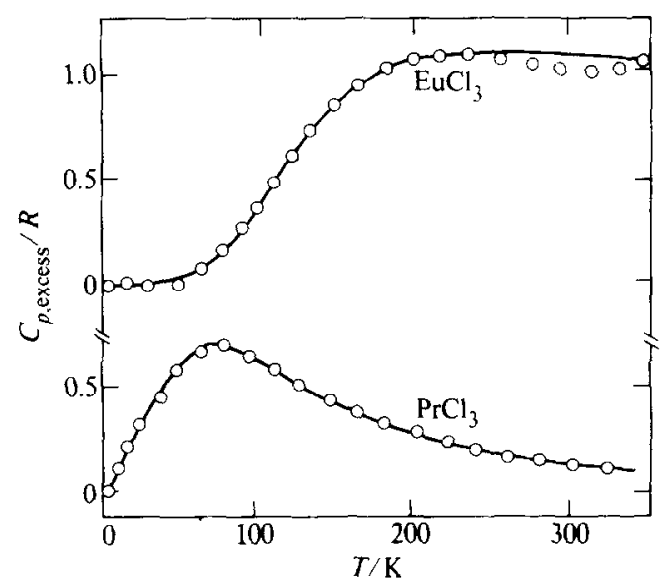

FIGURE 5. Schottky heat-capacities for two lanthanide trichlorides. - . The spectroscopic Schottky contributions: $O$, the calorimetric Schottky contributions resolved by the volumetric method. 
TABLE 3. Wavenumbers $\sigma$ for ${ }^{3} \mathrm{H}_{4}$ state of $\mathrm{PrCl}_{3}$

\begin{tabular}{|c|c|c|c|c|c|}
\hline \multicolumn{6}{|c|}{$\sigma / \mathrm{cm}^{1}$} \\
\hline $\mathrm{Pr}^{3+}$-doped $\mathrm{LaCl}_{3}$ & \multicolumn{5}{|c|}{ Concentrated $\mathrm{PrCl}_{3}$} \\
\hline $\begin{array}{l}\text { Absorption or } \\
\text { ffuorescence spectra }\end{array}$ & Calorimetry & CEF & ERS & $\mathrm{ERS}^{\prime \prime}$ & $\begin{array}{c}\text { Absorption } \\
\text { spectrid }\end{array}$ \\
\hline $0^{a}$ & $0^{h}$ & 0 & $0^{4}$ & $0^{e}$ & $0^{\prime}$ \\
\hline 33.1 & $\cdots$ & 29 & 30.5 & 32 & 31.8 \\
\hline 96.4 & $--\cdot$ & 99 & 99 & 100 & 99.6 \\
\hline 130.2 & 155 & 152 & 145 & 139 & \\
\hline 137.0 & 168 & 176 & 160 & & \\
\hline 199.1 & 235 & 230 & $(228)$ & $\cdots$ & \\
\hline
\end{tabular}

${ }^{a}$ Wavenumbers by absorption and/or fluorescence spectroscopy for $\mathrm{Pr}^{3{ }^{3}}$-doped $\mathrm{LaCl}_{3}$ (Sarup and Crozier) (53)

"Levels deduced from heat-capacities by volumetric lattice-contribution method. ${ }^{(2)}$

' Calculated from estimated crystal-field parameters. ${ }^{22,}$

'Observed in electronic Raman scattering (Chirico et al.). ${ }^{(2)}$

'Observed in electronic Raman scattering (Hougen and Singh).(4x)

Observed in absorption spectra (Dorman). ${ }^{154}$ )

\section{THE ROLE OF MASS}

The dashed and continuous curves of figure 6 represent $\left[C_{p}\left\{\mathrm{Gd}(\mathrm{OH})_{3}\right\}-\right.$ $\left.C_{p}\left\{\mathrm{La}(\mathrm{OH})_{3}\right\}\right]$ and $\left[C_{p}\left\{\mathrm{Y}(\mathrm{OH})_{3}\right\}-C_{p}\left\{\mathrm{La}(\mathrm{OH})_{3}\right\}\right]$ with the cooperative magnetic contribution to the $\mathrm{Gd}(\mathrm{OH})_{3}$ heat capacity deleted. The dotted curve is an estimate of $\left[C_{p}\left\{\mathrm{Lu}(\mathrm{OH})_{3}\right\}-C_{p}\left\{\mathrm{La}(\mathrm{OH})_{3}\right\}\right]$ derived by extrapolation of the experimentally observed lattice heat-capacity variation between $\mathrm{La}(\mathrm{OH})_{3}$ and $\mathrm{Gd}(\mathrm{OH})_{3}$ (e.g. see reference 19). If the lattice heat-capacity contribution for the lanthanide trihydroxides were exclusively a linear function of the molar volume, then the $\left[C_{p}\left\{\mathrm{Y}(\mathrm{OH})_{3}\right\}-C_{p}\left\{\mathrm{La}(\mathrm{OH})_{3}\right\}\right]$ curve would lie almost exactly midway between the dashed and dotted curves from 5 to $350 \mathrm{~K}$. In contrast, if the trend in the lattice contributions was determined principally by the molar-mass variation, it would lie entirely below the dotted curve instead of only for temperatures below about $120 \mathrm{~K}$.

\section{ACOUSTICAL AND OPTICAL LATTICE MODES}

From the results obtained for $\mathrm{Y}(\mathrm{OH})_{3}$, a shift in the relative importance of the cationic mass and volume in determining the trend in the lattice contributions across the series occurs near $100 \mathrm{~K}$. The experimental observations may be rationalized by considering the type and nature of the lattice vibrational modes being activated at each temperature. At very low temperatures, low-frequency modes-roughly characterized as unit-cell vibrations - are those primarily activated. The lanthanide contraction, an intramolecular contraction, has little effect upon these vibrations. In essence the force constants between the unit cells are unchanged, while the cell masses increase across the series. This occasions a decrease in the vibrational frequencies of the unit cells with increasing atomic number and, therefore, a corresponding increase in lattice contribution at a given temperature. 


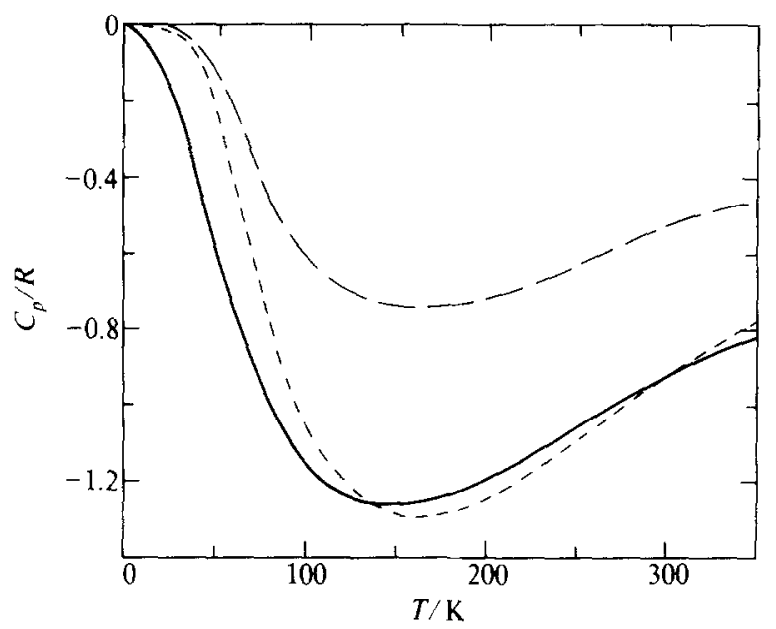

FIGURE 6. Differences in the heat capacities of three lanthanide trihydroxide pairs: - - - Gd-La: -, Y-La; $\cdots$, Lu-La.

At higher temperatures an increasing proportion of the observed heat capacity is due to thermal activation of optical vibrational modes. The effect of the lanthanide contraction upon these modes is to increase their frequency by increasing the intramolecular force constants to such an extent that the counteracting effect of the increased cationic mass is largely overshadowed. An analogous effect is routinely observed in temperature dependence of vibrational spectra. As the temperature is decreased (i.e. as the molecule contracts) the vibrational frequencies are generally seen to increase. In the case of the lanthanide trihydroxides the molar-mass variation between the lanthanum and gadolinium iso-anionic compounds is small enough to be insignificant in determining the trend in lattice contribution across the series between 10 and $350 \mathrm{~K}$. Even when considering $\mathrm{Y}(\mathrm{OH})_{3}$, which has a molar mass approximately $2 / 3$ that of the lanthanide compounds, the effect of molar mass is clearly dominant only below $80 \mathrm{~K}$. The difference between the heat capacities of electronically dilute lutetium and yttrium ethylsulfates ${ }^{(39,40)}$ clearly exhibits the same type of behavior as that of the corresponding trihydroxides.

The apparent differences with temperature of the functional dependence of the lattice contribution upon molar mass and volume makes it imperative that lattice approximations (e.g. the method of "corresponding states") which employ observations made at high temperatures to imply low-temperature properties or vice versa be applied with caution. Related problems have been discussed by Saxena, ${ }^{(29)}$ by Cantor, ${ }^{(30)}$ and by Kieffer. ${ }^{(31-35)}$

\section{ISO-ANIONIC LANTHANIDE SERIES AND GENERAL ISOSTRUCTURAL SERIES}

Thus the trend in lattice heat capacities of iso-anionic series of lanthanide compounds may be rationalized in terms of two contributing factors: molar mass and molar volume. At low temperatures, the lattice contribution is due primarily to thermal 
activation of acoustic lattice modes and molar mass is the dominant factor. At higher temperatures increasing thermal activation of optical lattice modes, which are strongly affected by the lanthanide contraction, results in lattice heat capacities which are related predominantly to the trend in molar volume. For the light lanthanide trihydroxides the molar-mass variation is dominated by the molar-volume effect at least above $50 \mathrm{~K}$. Only for much lighter $\mathrm{Y}(\mathrm{OH})_{3}$ is the mass effect clearly visible and then only below $100 \mathrm{~K}$.

Hence, in emphasizing the importance of volume, we do not mean to slight mass-especially not at lower temperatures. Results for $\mathrm{U}(\mathrm{OH})_{3}$, which is isostructural with the $\mathrm{Ln}(\mathrm{OH})_{3}$ 's, should help to clarify and to test the role of mass. Although we have demonstrated the great utility of the volumetric scheme as an interpolation device for $C_{p}(T)$ or $S(298 \mathrm{~K})$ for a system of isostructural compounds, what about the broader implications? How generally does it supplant the Latimer rule even when "augmented" to provide magnetic contributions, etc.?

We have examined isostructural series on which sufficient results exist to make a judgment. Many interesting trends are observed. For example, as seen in figure 7. extrapolation by the Latimer scheme from the entropies $(298 \mathrm{~K})$ of $\mathrm{MoS}_{2}, \mathrm{WS}_{2} . \mathrm{PtS}_{2}$ to that of $\mathrm{TiS}_{2}$ would lead to a Latimer-scheme value of $S / R=7$; on the other hand the volumetric approach would lead to $S / R=9.5$. Experiment $(S / R=9.4)$ confirms the latter. In other instances the general trend of cation mass with molar volume in iso-anionic series often tends to make choice between the two systems difficult inasmuch as molar mass and molar volume usually go hand in hand. Identification of key compounds on which to test the scheme and to develop more reliable correlations is underway. The broadening of these schemes has gone more slowly than anticipated since non-conformity often seems to signal an interesting excess contribution.

\section{Revisitation : the bixbyite sesquioxides}

It has been two decades since the initial heat-capacity measurements on the lanthanide sesquioxides were made in our laboratory. We were initially expecting

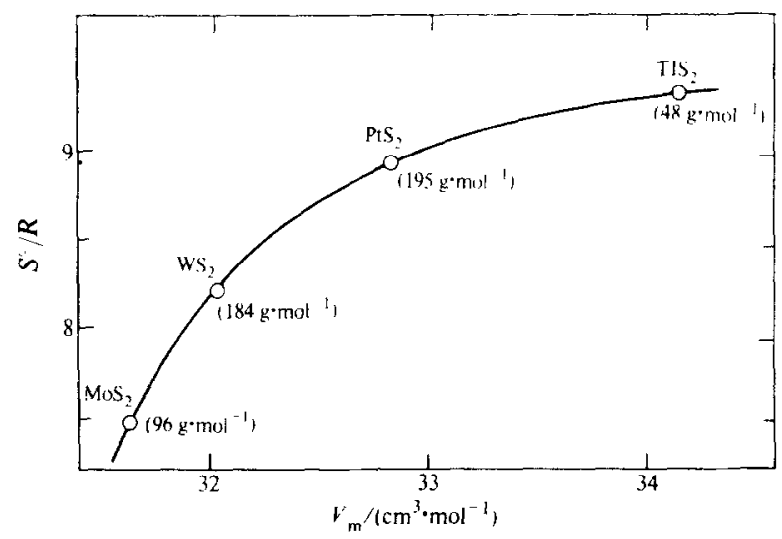

FIGURE 7. Correlation of entropies and molar volumes. The values in parentheses are molar masses of the cations. 
that Professor Dieke and his spectroscopic followers would soon put our results to a rigorous test. His death, however, brought much of this activity to an end. In the meantime, the lattice of the cubic sesquioxides has proven to be an excellent host material for some of the most powerful lasers built. ${ }^{(41-43)}$ In addition, over the last decade, lasers have greatly facilitated investigation of many low-probability nonlinear optical phenomena ${ }^{(44)}$ including especially vibrational and electronic Raman scattering $^{(45.46)}$ utilizing coherent, polarized, and tuneable excitation sources either through electronic Raman scattering and double-photon absorption of the crystalline-field states of lanthanide ions in sites with inversion symmetry. ${ }^{(46-48)}$ The earlier results that we took are very useful in testing the spectral assignments particularly when the volumetric approach to the lattice heat capacity is applied to the substances. A possible ambiguity in the interpretation of the spectral values arises from the fact that Raman-scattering experiments sometimes exhibit vibrational and electronic spectral peaks of similar magnitude. ${ }^{(47.49)}$ Moreover, some limitation in the resolution of lattice heat capacities also obtains. Together these restrictions preclude the total independence of the two approaches, but as will be shown, the final results are self-consistent. In addition, recent crystal-field calculations for ions in the $\mathrm{C}_{2}$ and $\mathrm{C}_{3 \mathrm{i}}$ sites in the $\mathrm{Ln}^{3+}$-doped $\mathrm{Y}_{2} \mathrm{O}_{3}$ provide an additional independent check of the experimentally deduced Stark splittings.

As an example of the modus operandi in the identification of the levels associated with $\mathrm{C}_{2}$ and $\mathrm{C}_{3 i}$ cation sites in the cubic (bixbyite) lattice, consider the observed crystalline electric field (CEF) splitting of the ground-state manifold [SL] $J$ for the ion $\mathrm{Er}^{3+}\left(4 \mathrm{fl}^{\mathrm{II}}\right)$ shown in table 4 . The experimental values listed in the second column are based on infrared results recently published. ${ }^{(24)}$ The identification of the levels is based on orientation-dependent studies, and they are in agreement with assignments reported elsewhere. The calculated Schottky contributions to the heat capacities

TABLE 4. Stark wavenumbers $\sigma$ for $\mathrm{Er}_{2} \mathrm{O}_{3}$ ground-state manifold $\left({ }^{4} I_{15 / 2}=[\mathrm{SL}] J\right.$-level $) \mathrm{C}_{2}$ and $\mathrm{C}_{3 i}$ sites

\begin{tabular}{|c|c|c|c|c|c|}
\hline & \multicolumn{3}{|c|}{$C_{2}$ sites } & \multicolumn{2}{|c|}{$C_{3 i}$ sites } \\
\hline & $\mathrm{Sp} .^{u}$ & Sch. ${ }^{\circ}$ & Calc: & Sp. ${ }^{d}$ & $\mathrm{~S}$ \\
\hline$Z_{n}$ & & & $\sigma / \mathrm{cm}^{-1}$ & & \\
\hline 1 & 0 & 0 & 0 & 0 & 0 \\
\hline 2 & 38 & 38 & 36 & 41 & 41 \\
\hline 3 & 75 & 75 & 73 & 80 & 80 \\
\hline 4 & 88 & 88 & 89 & 168 & 168 \\
\hline 5 & 159 & 159 & 166 & 328 & 328 \\
\hline 6 & 265 & 265 & 262 & 416 & 416 \\
\hline 7 & 490 & 490 & 485 & 485 & 485 \\
\hline 8 & 505 & 505 & 507 & 580 & 580 \\
\hline
\end{tabular}

\footnotetext{
assignments based on unpublished infrared data and verified from assignments and spectra.

${ }^{b}$ Wavenumbers used to calculate the Schottky contribution to the heat capacity appearing in figure 8 .

' Wavenumbers calculated using first-order perturbation theory as described in references 41 and 42 . Normalization of CEF parameters follow Wybourne's treatment of crystal-field theory in reference 51.

${ }^{d}$ Assignments based on unpublished electronic Raman scattering results
} 


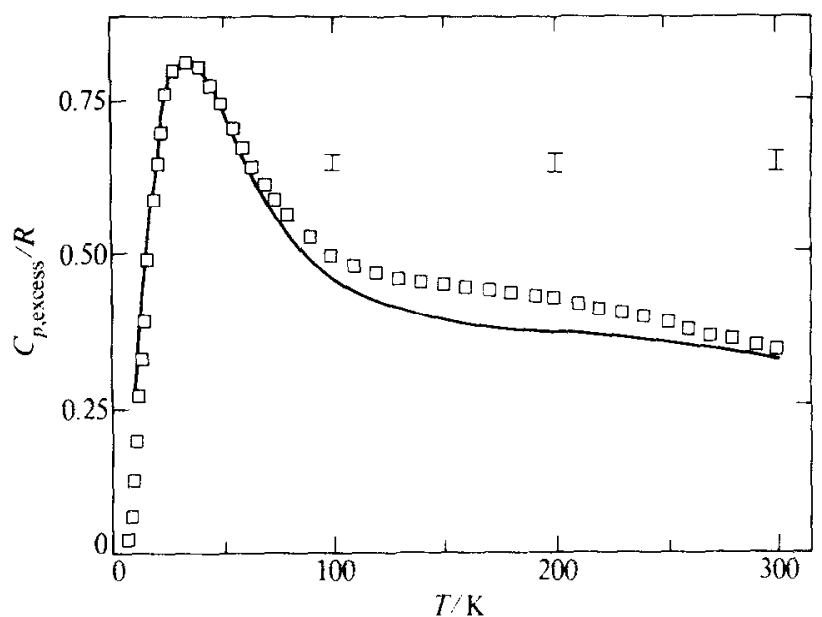

FIGURE 8. The Schottky contribution in $\mathrm{Er}_{2} \mathrm{O}_{3}$. - - Resolved calorimetric contribution $\square \square \square$. that based on spectroscopic levels in text.

(represented in figure 8) are based on the levels appearing in columns 3 and 6 in table 4. These values are identical to the levels identified from the various spectroscopic measurements as Kramers doublets associated with the CEF splitting of the ground-state manifold of the lanthanide ions in both sites. The excellent agreement between the spectroscopic and the thermal Schottky contributions to the total heat capacity lends assurance to the consistency of the assignments of the CEF levels. The positive deviations of the calorimetrically derived Schottky contributions at low temperatures from those calculated from the spectral quantities in both figures 8 and 9 are almost certainly occasioned by cooperative magnetic contributions. At higher temperatures the deviations rarely exceed the estimated experimental uncertainties. A further confirmation of such assignments may be found by

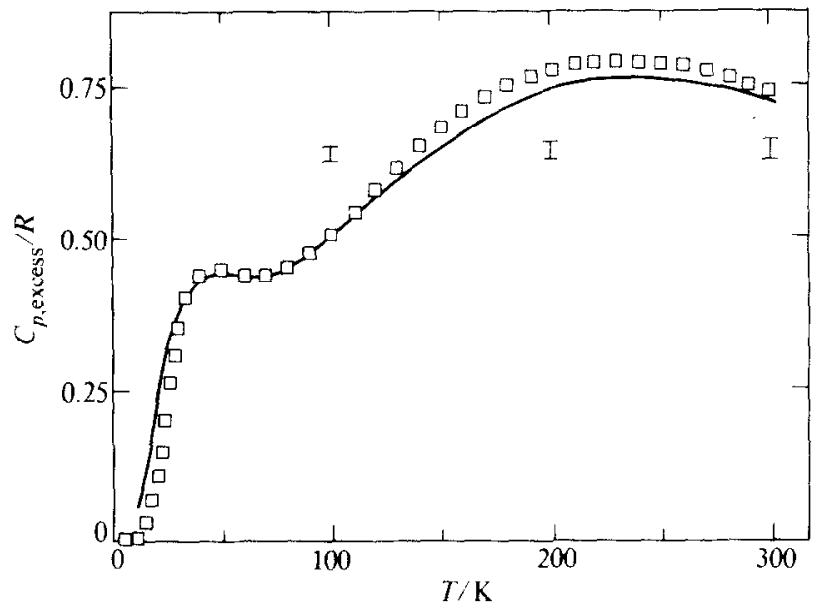

FIGURE 9. The Schottky contribution in $\mathrm{Dy}_{2} \mathrm{O}_{3}$. - - Resolved calorimetric contribution : - - .that based on spectroscopic levels in text 
comparing the recent crystal-field splitting calculations for these ions in both sites ${ }^{(50)}$ with the reported experimental splittings. The fourth column in table 4 shows the calculated CEF splitting for the lanthanide ions in the $C_{2}$ sites, and again excellent agreement is observed. The normalization of the CEF parameters follows Wybourne's treatment. ${ }^{(51)}$ In addition a point-charge and dipole lattice-sum calculation originally developed by Morrison and Leavitt ${ }^{(52)}$ has been extended to include calculations for ions in both sites for many levels. These calculations also substantiate the experimental assignments which we have made. We believe that the excellent agreement between spectroscopic quantities and heat-capacities relevant to the Schottky contributions will make it possible for interpretation or reinterpretation of existing and published e.p.r., Mössbauer, and heat-capacity results on these and other bixbyite sesquioxides. Figure 9 presents values for a similar analysis on $\mathrm{Dy}_{2} \mathrm{O}_{3}$ and again the general excellence of the agreement is very encouraging.

\section{The lanthanide sesquisulfides}

Our current interest has been centered intensively around the lanthanide sesquisulfides $\mathrm{Ln}_{2} \mathrm{~S}_{3}$, which represent a class of materials exhibiting unusual magnetic and semiconducting properties with many potential applications. These properties are manifestations of the $4 f$ states of the lanthanide ions which are accessible to cryogenic calorimetric, spectroscopic, and magnetic measurements. A brief summary of the very low-temperature (below $20 \mathrm{~K}$ ) measurements has been reported and a calorimetric, magnetic, and optical study ${ }^{(53)}$ of the $D y_{2} S_{3}$ crystal has also been reported. ${ }^{(54)}$ The authors, however, found that interpretation of most of the calorimetric results was complicated by possible magnetic ordering and crystal-field effects as well as by the lack of information on the lattice heat-capacity contribution. They endeavored to overcome such complications by using optical quantities to delineate the Stark levels and, therefore, the Schottky contribution. They then made estimates of the lattice contribution. Unfortunately, many of these studies are not based on adequately stoichiometric and well-characterized samples.

We have recently studied nine samples of lanthanide sesquisulfides prepared by the Ames Laboratory and carefully adjusted for stoichiometry; they were well characterized with respect to impurities, crystal structure, etc. Moreover, spectral studies and their interpretation are concurrently being made at Portland State University. Unfortunately, these sesquisulfides crystallize in four different structures and this makes difficult the interpolation of the lattice heat-capacity contribution for some of the compounds even by the utilization of the volumetric scheme. Many of the lower lanthanide sesquisulfides crystallize in the b.c.c. $\mathrm{Th}_{3} \mathrm{P}_{4}$ structure. ${ }^{\left({ }^{8}\right)}$ The infrared spectra on stoichiometric samples of lanthanum, cerium, and neodymium sesquioxides have been performed by several groups and the levels confirmed by recent reports of Raman scattering spectra. From the optical spectra analyzed and reported thus far, it is clear than $\mathrm{Ln}^{3+}$ in sesquisulfide systems is not found in local sites of octahedral or cubic symmetry even when the overall crystal structure has the $\mathrm{Th}_{3} \mathrm{P}_{4}$ b.c.c. defect-structure. Consequently, $\mathrm{Nd}^{3+}$ (and other $\mathrm{Kramers}$ lanthanide ions) will show splitting of the $J$-manifold into $(J+1 / 2) \mathrm{Kramers}$ doublets. 


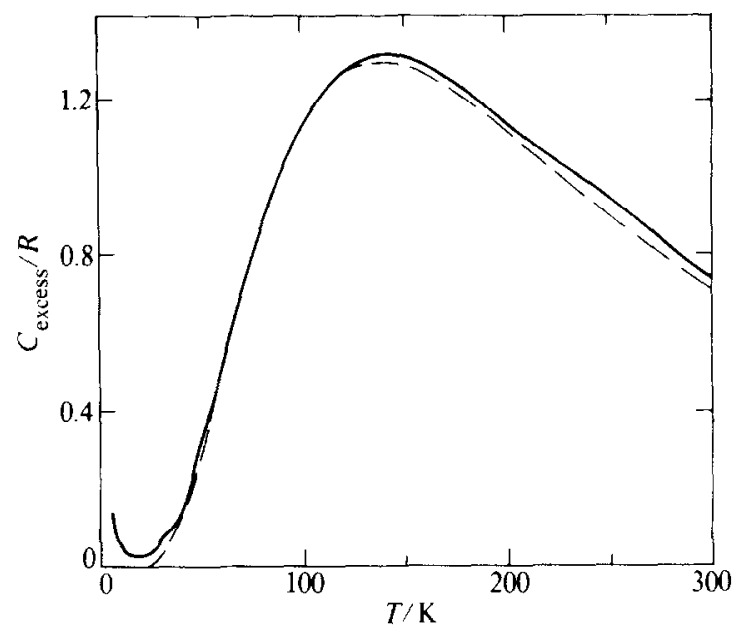

FIGURE 10. The Schottky contribution in $\mathrm{Nd}_{2} \mathrm{~S}_{3}$. - Resolved calorimetric contribution: - . . that based on spectroscopic levels in text.

Taking into account all of the available spectroscopic quantities and the results of lattice-sum calculation of neodymium sesquisulfide has led us to the (tentative) identification of the doublet levels of $0,76,140,190$, and $391 \mathrm{~cm}^{-1}$. The heat-capacity Schottky function defined by these points is shown in figure 10 and excellent agreement is observed over the peak part of the range.

A similar analysis on the $\mathrm{Tb}_{2} \mathrm{~S}_{3}$ crystal has (tentatively) led us to identify levels (with the degeneracies indicated in parenthesis following each level) of 0 (1). 4.8 (1). $24(1), 65(2), 130(2), 170(1), 185$ (1), 235 (2), and $300(2) \mathrm{cm}^{-1}$. Again the general excellence of the fit is to be seen in figure 11 .

We hope to study the remaining four sesquisulfides and to complete our interpretation of the entire family despite the difficulties occasioned by variations in the crystal structure and in the coordination numbers.

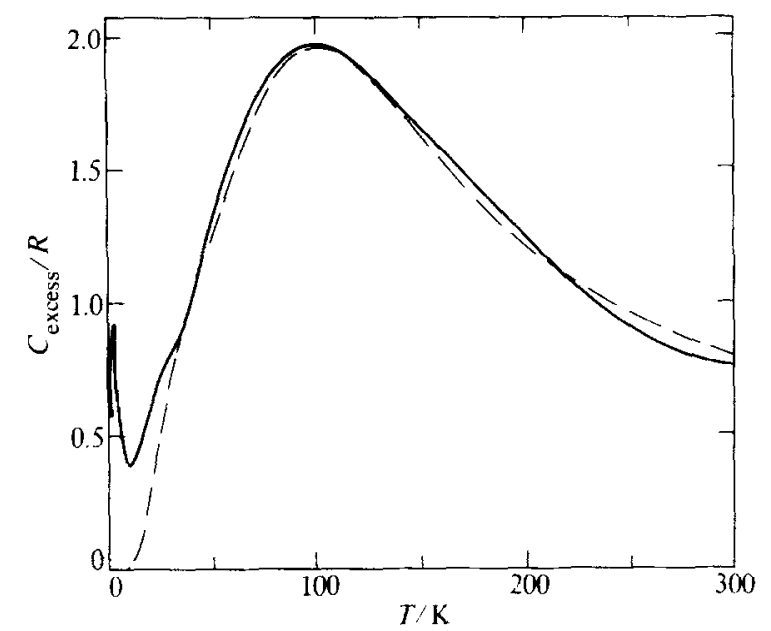

FIGURE 11. The Schottky contribution in $\mathrm{Tb}_{2} \mathrm{~S}_{3}$. - - Resolved calorimetric contribution: - - that based on spectroscopic levels in text. 
I should like to recognize (in addition to those authors already mentioned in the references) the current laboratory personnel who are working with me on the sesquisulfides. They are Professor Ramon Burriel (Zaragosa, Spain), Professor Magda El-Fass (Cairo, Egypt), Xiao-Xia Ma (Xi'an, People's Republic of China), and William Plautz. The collaboration of Vice President for Academic Affairs John Gruber (Portland, Oregon) and Dr Bernard Beaudry (Ames. Iowa) has been most encouraging. The author expresses his gratitude to the Thermodynamics and Structural Chemistry Section of the Chemistry Division of the National Science Foundation for its continuing support of the research endeavors on which this presentation is based under Grant CHE-8007977 and G EAR-8009538.

\section{REFERENCES}

1. Schottky, W. Phys. Z. 1922, 23, 448.

2. Westrum. E. F., Jr. unpublished results.

3. Meyer, P. H. E.; Colwell, J. H.; Shah, B. P. Am. J. Phys. 1973, 41. 332.

4. Justice, B. H.; Westrum. E. F., Jr. J. Phy's. Chem. 1963, 67, 339.

5. Justice. B. H.; Westrum, E. F., Jr. J. Phis. Chem. 1963, 67, 345

6. Justice, B. H.; Westrum, E. F., Jr. J. Phys. Chem. 1963, 67, 659.

7. Justice. B. H.; Westrum. E. F.. Jr.; Chang, E.; Radebaugh, R. J. Phys. Chem. 1969, $73,333$.

8. Justice, B. H.; Westrum, E. F.. Jr.; J. Phis. Chem. 1969, 73, 1959.

9. Penney, W. G. Phy's. Rev. 1933, 43, 2515.

10. Henderson, J. R.; Muramoto, M.; Gruber, J. B. J. Chem. Phys. 1967, 46, 2515.

11. Gruber. J. B.; Chirico. R. D.: Westrum. E. F., Jr. J. Chem. Phys. 1982, 76, 4600.

12. Sommers, J. A.; Westrum, E. F., Jr. J. Chem. Thermodynamics 1976, 8, 1115.

13. Sommers, J. A.; Westrum. E. F., Jr. J. Chem. Thermodynamics 1977, 9, 1.

14. Westrum, E. F., Jr. In Lanthanide/Actinide Chemistrv. Gould, R. F.: editor. Am. Chem. Soc.: Washington, D.C. 1967, p. 25.

15. Gronvold. F.; Westrum, E. F.. Jr. Inorg. Chem. 1962, 1. 36.

16. Stuttius, W. G. personal communication (1975); see Stuttius. W. G. Phys. Kondens. Mater. 1969, 10. 152.

17. Westrum, E. F., Jr.; Clever, H. L.: Andrews, J. I. S.; Feick. G. In Rare Earth Research III. Eyring, L. : editor. Gordon and Breach: New York. 1966, p.597; plus unpublished work.

18. Westrum, E. F.. Jr. Usp. Khim. 1979, 48, 2194.

19. Westrum. E. F., Jr. Russ. Chem. Rev. 1979, 48, 1186.

20. Latimer, W. M. J. Am. Chem. Soc. 1921, 43, 818 .

21. Latimer, W. M. J. Am. Chem. Soc. 1951, 73, 1480.

22. Westrum, E. F. Ir.; Chirico, R. D.; Gruher, J. B. J. Chem. Thermodrnamics 1980, 12, 717.

23. Chirico, R. D.; Westrum, E. F.. Jr.; Gruber. J. B.; Warmkessel. J. J. Chem. Thermodynamics 1979, 11. 835.

24. Chirico, R. D.: Westrum, E. F.. Jr. J. Chem. Thermodynamics 1980, 12, 71.

25. Chirico, R. D.; Westrum. E. F.. Jr. J. Chem. Thermodynamics 1980, 12, 311.

26. Chirico, R. D.; Westrum. E. F., Jr. J. Chem. Thermodynamics 1981, 13, 519.

27. Chirico. R. D.; Westrum. E. F., Jr.; Boerio-Goates, J. J. Chem. Thermodynamics 1981, 13, 1087.

28. Westrum, E. F., Jr. In The Rare Earths in Modern Science and Technolog. Vol. 2. McCarthy, G. J.; Rhyne, J. J.: Silber, H. B.: editors. Plenum Press: New York. 1980, p. 371.

29. Saxena, S. K. Science 1976, 193, 1241

30. Cantor, S. Scionce 1977, 198, 206.

31. Kieffer. S. W. Rev. Geophys. Space Phys. 1979, 17. 1.

32. Kieffer, S. W. Rev. Geophys. Space Phys. 1979, 17, 20.

33. Kieffer, S. W. Rev. Geophys. Space Phis. 1979, 17. 35.

34. Kieffer. S. W. Rev. Geophls. Space Phys. 1979, $17,862$.

35. Kieffer, S. W. Rev. Geophys. Space Phi's. 1982, $20,827$.

36. Cone, R. L.; Faulhaber, R. J. Chem. Phys. 1971, 55, 5198

37. Reference 23 together with unpublished spectra cited in table 1 therein.

38. Scott. P. D.; Meissner, H. E.; Crosswhite, H. M. Phy's. Lett. A 1969, 28, 489. 
39. Gerstein, B. C.; Jennings, L. D.; Spedding, F. H. J. Chem. Phys. 1962, 37, 1496.

40. Gerstein. B. C.; Penny, C. J.; Spedding, F. H. J. Chem. Phys. 1962, 37, 2610.

41. Dieke, G. H. Spectra and Energy Levels of Rare Earth lons in Crystals. Interscience: Ncw York. 1968.

42. Hufner, S. Optical Spectra of Transparent Rare Earth Compounds. Academic Press: New York. 1978.

43. Reisfeld, R.; Jørgensen, C. K. Lasers and Excited States of Rare Earths. Springer: Berlin. 1977.

44. DiBartolo, B. Optical Interactions in Solids. Wiley: New York. 1968.

45. Yariv, A. Quantum Electronics, Wiley: New York. 1967.

46. Axe, J. D. Phys. Rev. A. 1964, 42, 136; see also Elliott, R. J.; Loudon, R. Phys. Lett. 1963, $3,189$.

47. Gruber, J. B. In Proceedings of the 9th Rare Earth Research Conference, Blacksburg, Virginia. NTIS, U.S. Dept. of Commerce: Washington. D.C. 1971, p. 465.

48. Hougen, J. J.: Singh, S. Proc. R. Soc. London A 277. 1964, 193; see also Phy's. Rev. Lett. 1963, 10.406.

49. Lejus, A. M.; Michel, D. Phy's. Status Solidi 1977, 84, K105.

50. Gruber, J. B.; Chang, N. C.; Leavitt, R. P.; Morrison, C. A. to be published.

51. Wybourne. B. G. Spectroscopic Properties of Rare Earths. Interscience: New York. 1965.

52. Morrison, C. A.; Leavitt, R. P. J. Chem. Phys. 1979, 71, 2366.

53. Sarup, R.: Crozier, M. H. J. Chem. Phys. 1965, 42, 371.

54. Dorman. E. J. Chem. Phys. 1966, 44, 2910. 